\title{
Patrones espacio-temporales de mortalidad por frío excesivo en México
}

\section{Spatio-temporal patterns of mortality by excessive cold in Mexico}

\begin{abstract}
David Maximiliano Zermeño-Díaz* Leticia Gómez-Mendoza*

Rodolfo Acuña-Soto*

Recibido: abril 12 de 2020.

Aceptado: agosto 21 de 2020.

\section{Resumen}

Este estudio presenta una caracterización espacio-temporal de la relación entre el clima y la mortalidad por frío excesivo usando registros con 37 años de información (1979-2015). Se analizaron datos de mortalidad, datos poblacionales de cuatro censos nacionales y datos en malla de temperaturas mínimas mensuales usando histogramas, correlaciones y un análisis de tendencias. La mortalidad más alta encontrada ocurre principalmente en cuatro regiones relativamente frías: el noroeste de México, el Altiplano Central, Los Altos de Chiapas y la frontera norte de México. Aunque la mayoría de nuestros resultados son consistentes con el paradigma "a más frío, más muertes por frío intenso", otros sugieren una relación compleja entre la mortalidad en tasas y las bajas temperaturas. En particular, se encontró una correlación débil entre tasas de mortalidad y temperaturas mínimas, y tendencias de tasas de mortalidad positivas en medio de un clima con tendencias cálidas. Estos resultados sugieren que la aclimatización a un clima más cálido podría incrementar la vulnerabilidad de las personas a eventos extremos fríos, lo cual, consecuentemente, sugiere que, aun bajo un entorno futuro más cálido en México, serían necesarias medidas de adaptación ante el frío intenso basadas en diagnósticos de vulnerabilidad.
\end{abstract}

Palabras clave: mortalidad por frío, tendencias, caracterización de la vulnerabilidad.

\begin{abstract}
This study presents a Spatio-temporal characterization of the relation between climate and mortality by intense cold using 37 years of data (1979-2015). It was analyzed mortality data, data from four national censuses, and gridded monthly minimum temperatures. These datasets were analyzed using frequency diagrams, and correlation and trend analyses. The highest mortality found mainly occurs in four relatively cold regions: the northwest of Mexico, the Mexican plateau, Los Altos de Chiapas, and the north border of Mexico. Although most of our results are consistent with the "the colder it is, the higher the mortality" paradigm, other results suggest a more complex relation between mortality rates and low temperatures. Particularly, it was found a weak correlation between mortality rates and low temperatures, and positive trends in mortality rates within warm climates with warm trends. These results suggest that acclimatization to a warmer climate might increase the vulnerability to extreme cold events. If so, even under a future warmer climate, adaptation strategies to intense cold in Mexico would be needed; such strategies would ideally be based on diagnostics of vulnerability.
\end{abstract}

Keywords: cold mortality, trends, vulnerability characterization.

*Universidad Nacional Autónoma de México, México. Correos electrónicos: davidzermeno@filos.unam.mx, gomez.leticia852@gmail.com, acunasoto.rodolfo@gmail.com,gomez.leticia852@gmail.com, acunasoto.rodolfo@gmail.com 


\section{Introducción}

Una de las preguntas más significativas relacionadas con el cambio climático es aquella relativa a sus impactos en la salud humana (Githeko y Woodward, 2003; IPCC, 2012): ¿Cómo un clima cada vez más cálido puede incrementar padecimientos sanitarios? La respuesta es relativamente compleja, porque los impactos del clima dependen, entre otros factores, de la vulnerabilidad de la población (Riojas Rodríguez et al., 2006). En particular, para el caso de los impactos del frío excesivo se esperaría una disminución de impactos (Compeán, 2013; McMichael et al., 2008). Sin embargo, existe incertidumbre en el tema, ya que la vulnerabilidad de las personas al frío es compleja y dinámica (Medina-Ramon y Schwartz 2007; Milojevic et al., 2016; Singh et al., 2019).

La importancia de ambos elementos del problema -una amenaza climática y una vulnerabilidad social- subraya que se deben tratar los impactos del frío intenso mediante el enfoque de la gestión del riesgo; es decir, basado en caracterizaciones objetivas de amenazas y vulnerabilidades (Cardona, et al., 2012; De Sherbinin, Schiller y Pulsipher, 2007; Vargas y Magaña, 2020).

\section{Marco teórico}

En México, existen relativamente pocos estudios que aplican la perspectiva del riesgo para analizar los impactos del frío extremo en la población (Cohen y Dechezleprêtre, 2017; Compeán, 2013; Jáuregui, Sánchez y Cabañas, 2020; McMichael et al., 2008); entre los más completos destaca el de Jáuregui, et al. (2020), pues se analizó la mortalidad por frío excesivo en conjunto con otras causas de origen socio-natural (calor intenso, tormentas, terremotos, entre otros). Asimismo, se encontró que para el periodo 2000-2015 la mortalidad por frío excesivo tiene una tendencia negativa, pero se sitúa como segunda muerte por eventos hidrometeorológicos en México. Del total de víctimas por frío intenso, el sector poblacional masculino representó casi el 89\%; en promedio, personas de 40 años de edad; la mayoría de estas muertes ocurrió en áreas metropolitanas (ciudades donde hay más de medio millón de habitantes).

Aunque puede considerarse el enfoque de la gestión del riesgo como el marco teórico para el análisis de mortalidad por frío excesivo, pocos estudios han tratado el tema incluyendo de manera directa parámetros que describan la amenaza de las bajas temperaturas medioambientales (Compeán 2013; McMichael et al., 2008), ya que la mayoría se ha centrado en las estadísticas de mortalidad y de vulnerabilidad por grupos demográficos y económicos. Tal escasez de estudios podría estar parcialmente originada por la dificultad para evaluar "frío excesivo" - ¿qué tanto es excesivo?- y para evaluar indicadores cuantitativos de vulnerabilidad (Magaña, 2013). 
Si caracterizar la amenaza presenta dificultades, caracterizar la vulnerabilidad es una tarea más compleja debido a que no existe un conjunto de indicadores universalmente aceptados para medir la vulnerabilidad (Blaikie et al., 1996; IPCC, 2012). Sin embargo, para el caso particular de los impactos del frío excesivo, la mortalidad es un indicador con cierto grado de objetividad como se ha demostrado en estudios previos (Jáuregui, Sánchez y Cabañas, 2020; McMichael et al., 2008).

La vulnerabilidad al frío excesivo natural en México está íntimamente relacionada con la pobreza; al analizar datos de mortalidad por exposición al frío excesivo durante el periodo 1998-2010, Cohen y Dechezleprêtre (2017) descubrieron que en México una persona pobre tiene una probabilidad cuatro veces más alta de fallecer que una persona acaudalada. También encontraron que las personas con un ingreso menor al de la media nacional son más propensas a morir por frío, y mostraron que el acceso a los servicios de salud, en este caso el programa gubernamental "Seguro Popular" (Knaul et al., 2012), estuvo correlacionado con una reducción en las muertes por frío natural excesivo entre la población de los estratos más pobres en casi un 30\% (este análisis usó datos de 2004 a 2010).

\section{Motivación y objetivos}

Dado un problema complejo, la gestión del riesgo provee un enfoque estructurado para el diseño de medidas de adaptación y reducción del desastre. Sin embargo, este enfoque comienza con caracterizaciones cuantitativas de cada uno de los elementos involucrados (Neri y Magaña 2016), las cuales tratan de representar a éstos de la forma más completa posible, usando los parámetros más confiables disponibles (Magaña, 2013). Con tal idea como motivación, este estudio presenta un acercamiento al asunto de los impactos del frío extremo en México desde la perspectiva del riesgo. En particular, tiene como objetivo principal construir una caracterización básica de la relación espacio-temporal que existe entre el clima (amenaza) y la mortalidad por frío excesivo (vulnerabilidad) en México.

\section{Datos y metodología}

En este estudio se analizaron 37 años, periodo1979-2015, de registros de mortalidad por frío excesivo, número de habitantes y datos en malla de temperaturas mínimas mensuales. La fuente primaria de información en México sobre mortalidad son los certificados de defunción expedidos por médicos o personal acreditado por la autoridad sanitaria (médicos forenses, médicos rurales, etcétera).

Una vez elaborado y firmado el Certificado de Defunción, se procede al trámite para obtener el Acta de Defunción, que es un documento obligatorio. Esto se realiza ante las Oficialías del Registro Civil, aun cuando, en su caso, interviene el Ministerio Público. Las oficinas concentradoras del Registro Civil estatales envían mensualmente la información al 
Instituto Nacional de Geografía e Informática (INEGI) en donde se organiza y codifica; después, se manda una copia a la Secretaría de Salud. Los datos resultantes son mensuales e incluyen edad, sexo y municipio de residencia al momento de la defunción. La información es pública y se puede encontrar en los sitios web tanto del INEGI (INEGI, 2019) como de la Dirección General de Información en Salud (GDIS, 2020), de la Secretaría de Salud.

Siguiendo la Ley Federal de Protección de Datos Personales, la información de mortalidad se anonimiza y se presenta en forma final de manera mensual y municipal (INEGI, 2011). Para este estudio, los datos de mortalidad fueron obtenidos del Sistema Nacional de Información en Salud (SINAIS, 2018), actualmente denominado DGIS.

Los datos de mortalidad ocupados en esta investigación corresponden a los de la clave 901 (muerte por exposición al frío natural excesivo) de la versión 9 de la Clasificación Internacional de Enfermedades y Problemas Relacionados con la Salud (CIE) de la Organización Mundial de la Salud (OMS, 2020), y bajo la clave X31 (muerte por exposición al frío natural excesivo) de la CIE versión 10. Se ocuparon dos claves de dos versiones de la CIE, ya que cada una corresponde a un periodo diferente: la 901 para 1979-1997 y la X31 para 1998-2015.

La CIE es un sistema de información sustentado por un acuerdo de la asamblea de la OMS, donde México es solidario. Tiene como propósito unificar las normas para el estudio de las tendencias, estadísticas y criterios para la administración de salud en todos los países miembros. Es la nomenclatura estándar empleada actualmente por los sistemas de salud y administradoras de seguros públicos y privados en todo el mundo.

Por otro lado, se usaron los datos de temperatura mínima mensual $\left(T_{\min }\right)$ de la base TS4.01 del Climate Research Unit (CRU, 2019). Estos datos tienen una resolución temporal mensual y una resolución espacial de $0.5^{\circ} \mathrm{X} 0.5^{\circ}$ (aprox. $111.12 \mathrm{~km}{ }^{2}$ ), y fueron elegidos para este estudio porque se consideran una referencia estándar (e. g., Tabor y Williams, 2010).

La relación mortalidad-clima se analizó desde dos perspectivas: su correlación (Pearson) y la coincidencia de patrones espaciotemporales mediante mapas y diagramas de frecuencia. Asimismo, se estudiaron tendencias lineales, las cuales fueron calculadas con el método de ajustes lineales por mínimos cuadrados (se toma el valor de la pendiente del ajuste) (Santer et al., 2000). La significancia estadística de las correlaciones y tendencias lineales calculadas fueron probadas al 95\% de confiabilidad $(a=0.5)$ asumiendo una distribución $t$-Student de dos colas.

Los datos de CRU TS4.01 fueron comparados con los de mortalidad en términos de los números totales de personas fallecidas y de tasas de mortalidad. Para calcular éstas (por cada 100,000 habitantes), se ocuparon los datos de número de habitantes por municipio de los censos de 1970, 1980, 1990, 2000, 2010 y del conteo 2015 (INEGI, 2010). Después, para 
comparar directamente los datos de tasas de mortalidad con los de clima, la información de los censos de cada municipio fue interpolada a una resolución mensual, debido a que los datos de mortalidad y de clima son mensuales, pero los de número de habitantes son decadales (de los censos de 1980 a 2010) y quinquenales (los del censo de 2015).

El método de interpolación usado fue el estándar de mínimos cuadrados. Con ambas bases de datos en resolución mensual, se obtuvieron las tasas de mortalidad al dividir el número de muertes municipales de cada mes por el número de habitantes estimados para ese mes, de ese municipio. Finalmente, el resultado fue multiplicado por 100,000. Los detalles más específicos sobre los métodos empleados, incluyendo los diagramas de frecuencias empleados, se discuten junto con sus resultados en la siguiente sección.

\section{Resultados}

Patrones temporales

Durante el periodo analizado, se registraron un total de 1,207 muertes por frío natural excesivo; de las cuales, 801 (64.2\% del total) ocurrieron en los meses de invierno (diciembre a febrero), bajo temperaturas en mínimas mensuales menores a $5^{\circ} \mathrm{C}$ (cuadro 1), y $127(10.4 \%)$ en verano (junio a septiembre), bajo temperaturas por arriba de $14^{\circ} \mathrm{C}$.

Cuadro 1. Total de casos mensuales de mortalidad por frío natural excesivo

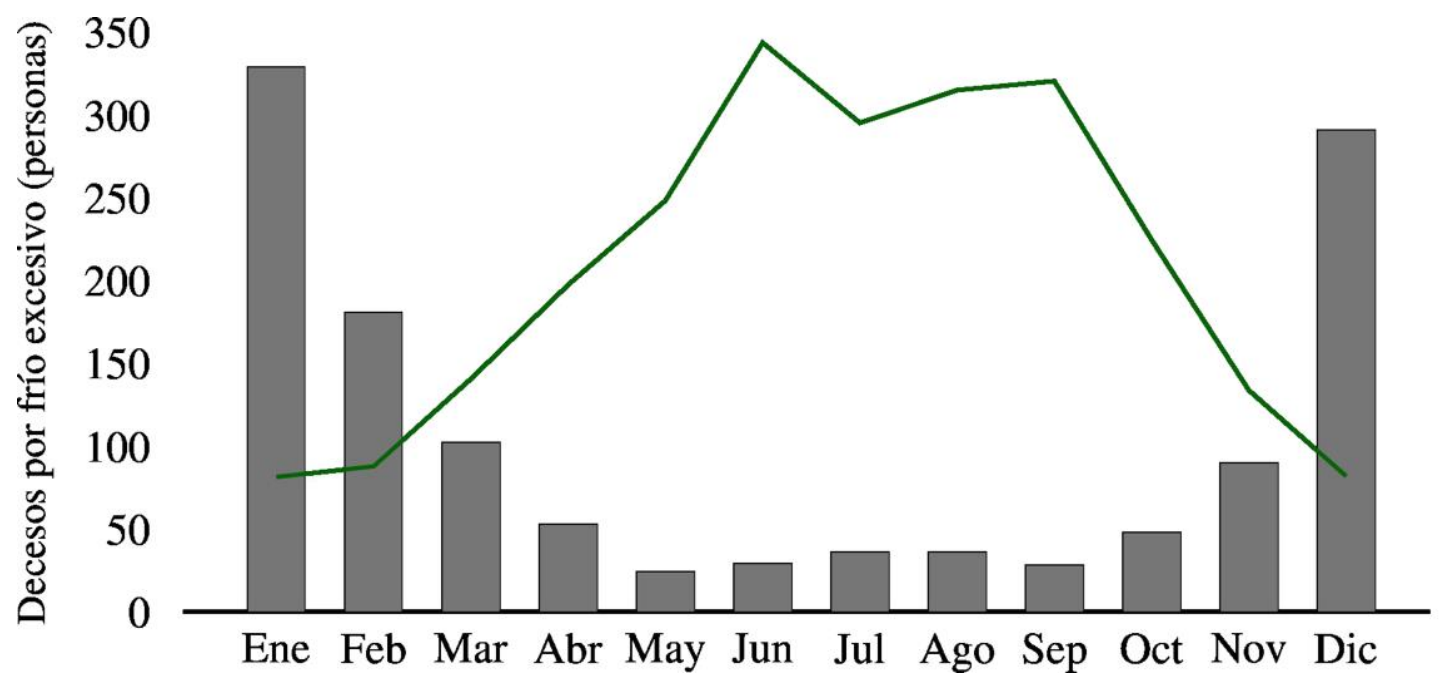


La correlación entre tasas de mortalidad y temperatura mínima mensual arrojó una asociación no significativa de -0.12. Este resultado contrasta con la marcada estacionalidad mostrada en el cuadro 1 y con las relaciones empíricas halladas por Compeán (2013) que asocian la mortalidad por frío con variables climáticas. Para explorar la causa de esta débil correlación, realizamos un análisis de frecuencias. Para ello, primero ordenamos datos de mortalidad y población según la temperatura mínima; usamos amplitudes de categoría de $1^{\circ} \mathrm{C}$ (ver eje horizontal del cuadro 2 ).

Así, a cada muerte por frío se le asoció la temperatura del mes en la que ocurrió, y el tamaño de su respectiva población municipal. Con esa información calculamos la frecuencia de mortalidad y el tamaño de población promedio en cada categoría de temperatura mínima. Los resultados de este análisis muestran (cuadro 2) que aproximadamente dos terceras partes de muertes por frío ocurren bajo temperaturas mínimas mensuales inferiores a $8^{\circ} \mathrm{C}$ (a la izquierda de la línea azul discontinua que marca el percentíl 67 de temperatura mínima; cuadro 2), pero dos terceras partes de los datos poblacionales se encuentran por debajo de $18^{\circ} \mathrm{C}$ (a la izquierda de la línea verde discontinua que marca el percentíl 67 de población media; cuadro 2). La diferencia de $10^{\circ} \mathrm{C}$ entre la línea que marca dos terceras partes de muertes por frío y dos terceras partes de población media muestra que el factor poblacional -a mayor población, mayor mortalidad- no es suficiente para explicar todas las muertes por frío.

Por lo tanto, se requiere incluir un factor climático -a más temperaturas más bajas, mayor mortalidad-. Sin embargo, tampoco es suficiente para establecer una relación determinista $(X:$ temperatura $\rightarrow Y$ : mortalidad) mediante una correlación lineal (hayamos una correlación de -0.12). Estos resultados sugieren que un enfoque más apropiado para analizar el problema es el probabilista, característico de la gestión del riesgo: las probabilidades de que ocurra $Y$ : alta mortalidad dado $X_{1}$ : bajas temperaturas son significativamente más altas que dado $X_{2}$ : altas temperaturas.

Mientras que una alta mortalidad por temperaturas bajas es predecible, la relativa alta mortalidad durante verano (casi 10\%; cuadro 1) es sorpresiva. Sin embargo, este resultado poco intuitivo podría explicarse al menos por dos factores no considerados en este estudio: vulnerabilidad médica de las personas fallecidas al frío y la ocurrencia de eventos extremos no manifestados en las temperaturas mínimas mensuales que analizamos (e. g., en resoluciones temporales diarias). 
Cuadro 2. Distribución de frecuencias de muertes por frío natural excesivo ordenados según la temperatura del mes en el que ocurrieron (línea continua azul oscuro; su eje está a la derecha) y promedios de cantidad de población de los municipios donde se registraron las muertes por frío (barras verde claro; su eje está a la izquierda)

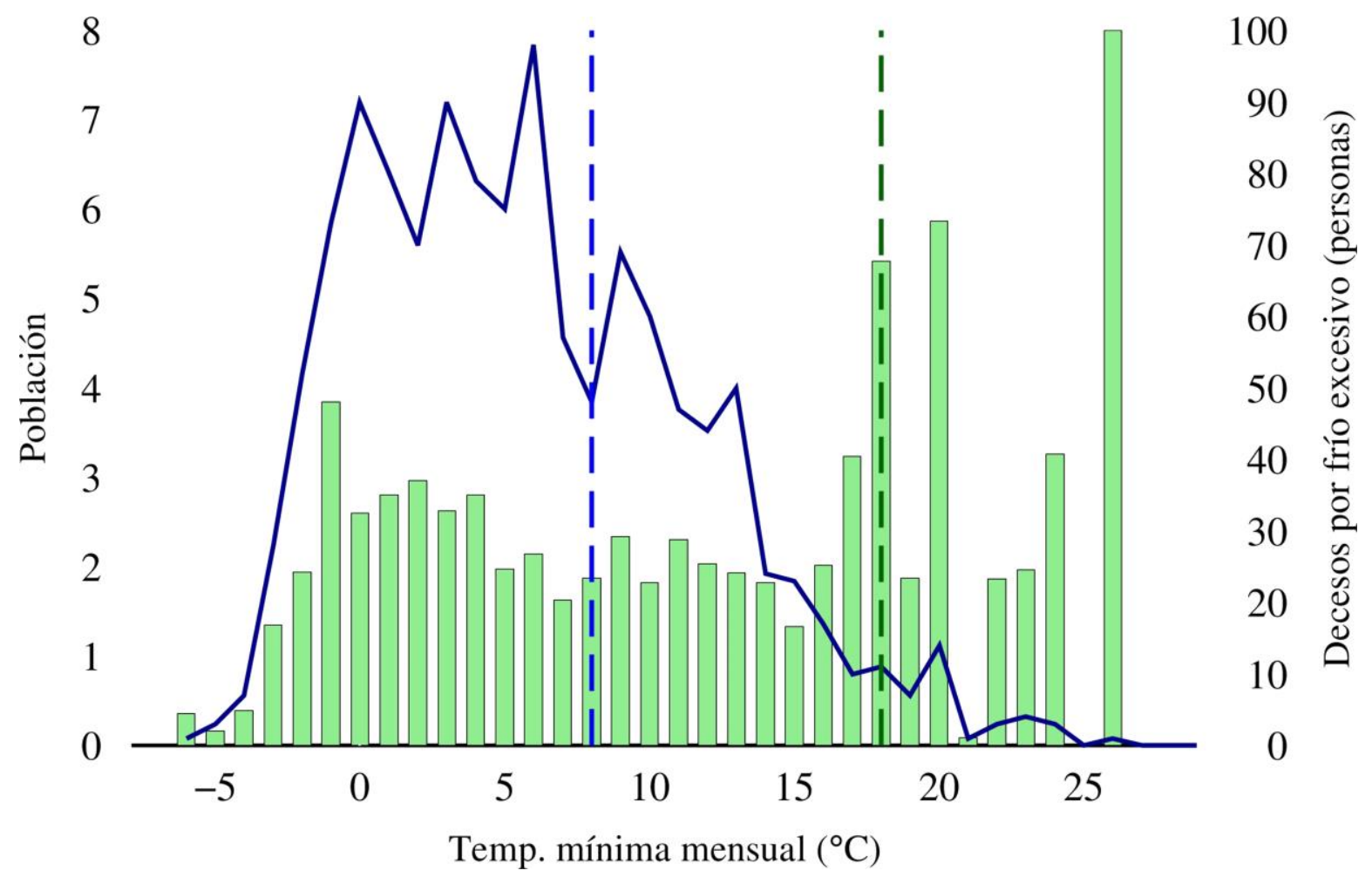

Nota: la línea discontinua azul $\left(a 8^{\circ} \mathrm{C}\right.$ ) marca el percentíl 67 de los datos de mortalidad y la línea verde discontinua (a $18^{\circ} \mathrm{C}$ ) marca el percentíl 67 de los datos de población.

Fuente: elaboración propia.

\section{Patrones demográficos}

La población en México ha seguido un incremento lineal durante el periodo de estudio (cuadro 3a), pero la tendencia en casos de mortalidad ha seguido una trayectoria discontinua (cuadro 3b). La evolución temporal de la mortalidad por frío natural excesivo en México podría dividirse en tres periodos (sólo con fines ilustrativos): de 1979 a 1993, de 1994 a 2003 y de 2004 a 2015. En el primero, la mortalidad se mantuvo aproximadamente constante, con totales que van de los 5 a los 30 casos cada año.

En el segundo periodo, la mortalidad tuvo una marcada tendencia positiva: el número de casos por año se elevó de 13 hasta casi 70. En la tercera, la mortalidad tuvo una tendencia negativa débil: el número de casos por año se redujo de casi 70 a 38, pero durante el periodo en el que esto ocurre se presentaron fluctuaciones relativamente grandes. La evolución de estas tendencias es similar si se evalúa en términos de tasas (cuadro 3c), y es consistente con lo documentado por Jáuregui et al. (2020) para el periodo 2000-2015. 
Cuadro 3. Distribución temporal de (a) la población total anual en México y de la mortalidad por frío intenso en términos de (b) cantidad de personas y de (c) tasas de mortalidad
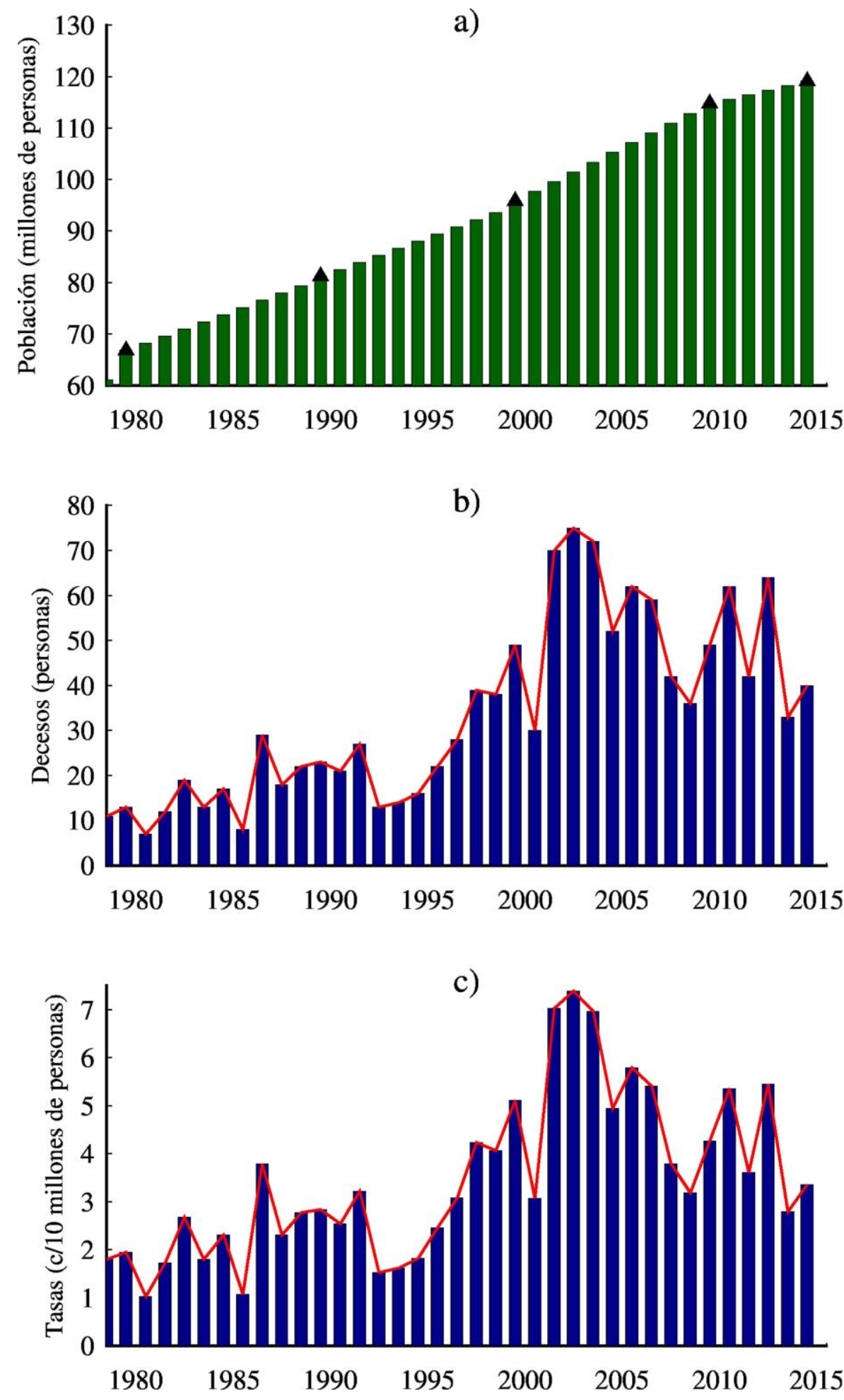

Nota: la línea roja se muestra como ayuda visual en la evolución de la mortalidad; los triángulos indican datos provenientes de un censo o un conteo (ausencia de triángulo son interpolaciones). 
Del total de muertes por frío registradas de 1979 a 2015 (1,207), cerca del 15\% fueron mujeres (cuadro 4a) y un poco más del $78 \%$ fueron hombres (cuadro $4 \mathrm{~b}$ ) de 49 años en promedio, lo cual, en general, es consistente con Jáuregui et al., (2020), pues encontraron estadísticas similares para el periodo 2000-2015.

Por otro lado, la mayor mortalidad entre mujeres adultas ocurre alrededor de los 85 años. Del cuadro 3b, también es notable que los niños y las niñas menores de 5 años registran valores altos de mortalidad. Las tendencias lineales para cada grupo poblacional mostrado en el cuadro 4 son estadísticamente significativas en el grupo de hombres (cuadro 5), lo cual revela que en las últimas tres décadas hay una tendencia al alza de mortalidad por frío entre hombres de entre 50 y 80 años de edad. Estos resultados son consistentes con estudios previos nacionales (Jáuregui et al., 2020) y de otros países (Nixdorf-Miller, Hunsaker y Hunsaker, 2006; Taylor, et al. 2001) que han mostrado una disminución en la mortalidad en todos los grupos de edad.

\section{Cuadro 4. Distribuciones de frecuencias por edad (cada 5 años) y por sexo (a, b) de casos de mortalidad por frío natural excesivo}

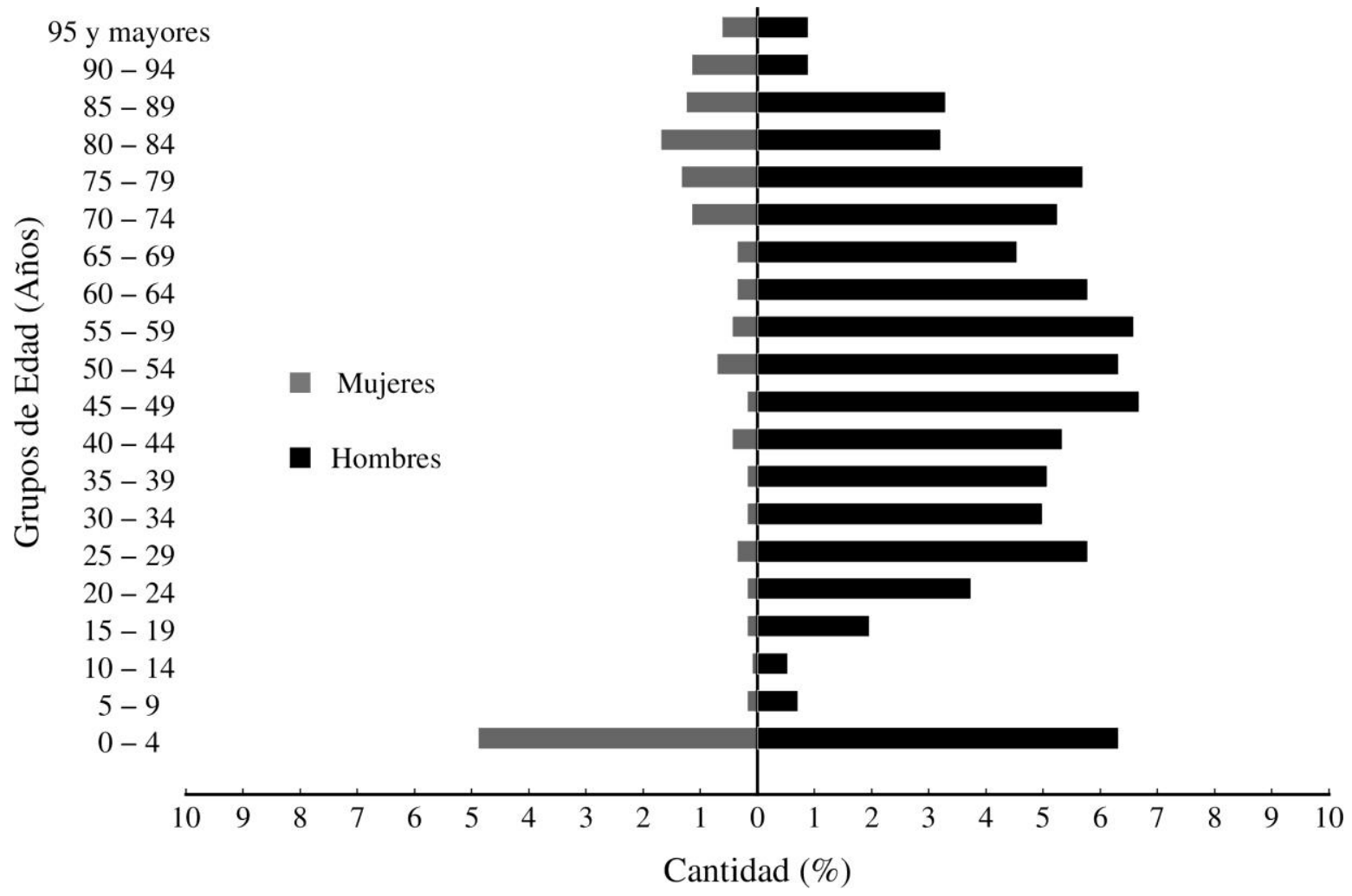

Fuente: elaboración propia. 
Cuadro 5. Tendencias (1979-2015) de tasas de mortalidad (cada 100,000 hab.) por frío natural excesivo por grupos de edad; las tendencias estadísticamente significativas son indicadas con un cuadro



\section{Tendencias (casos c/100,000 hab. de 1979-2015)}

Fuente: elaboración propia.

\section{Patrones espaciales}

La distribución espacial de mortalidad total (cuadro 6a) puede dividirse en cuatro regiones: 1) el noroeste de México, a barlovento de la Sierra Madre Occidental, principalmente sobre Sonora, sur Chihuahua y noroeste de Durango; 2) el Altiplano Central sobre su lado oriente, con límites en la frontera con Sierra Madre Oriental; 3) la frontera con Estados Unidos; y 4) el sureste de México sobre Los Altos de Chiapas. De esta distribución espacial de mortalidad, tres subregiones tienen los más altos índices de pobreza a nivel nacional, de entre 43\% y 82\% (mapa 2.1 en PNUD, 2019), y estas mismas al noroeste están caracterizadas por ser regiones montañosas; estás tres regiones son el sur de Chihuahua y noreste de Durango, Los Altos de Chiapas y el lado oriente del Altiplano Central. 
Cuadro 6. Temperatura mínima promedio con (a) las muertes por frío intenso incluyendo todos los grupos demográficos, (b) incluyendo sólo el grupo de muertes de mujeres, (c) sólo el de hombres, (d) y sólo el de menores a 5 años

a)

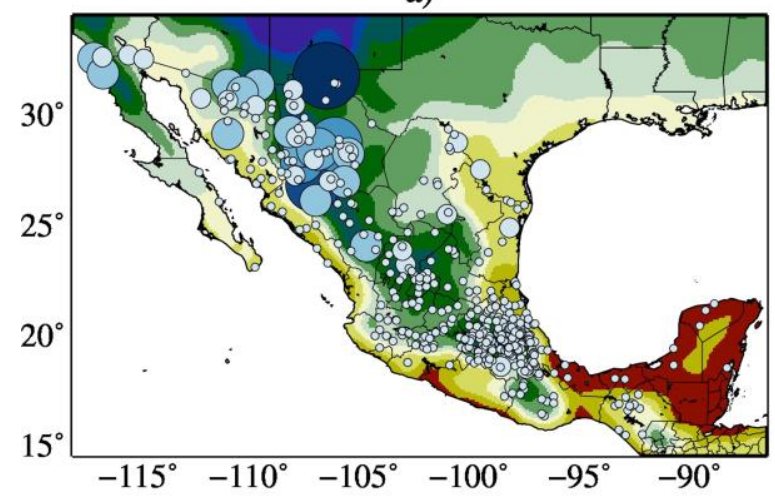

c)



b)

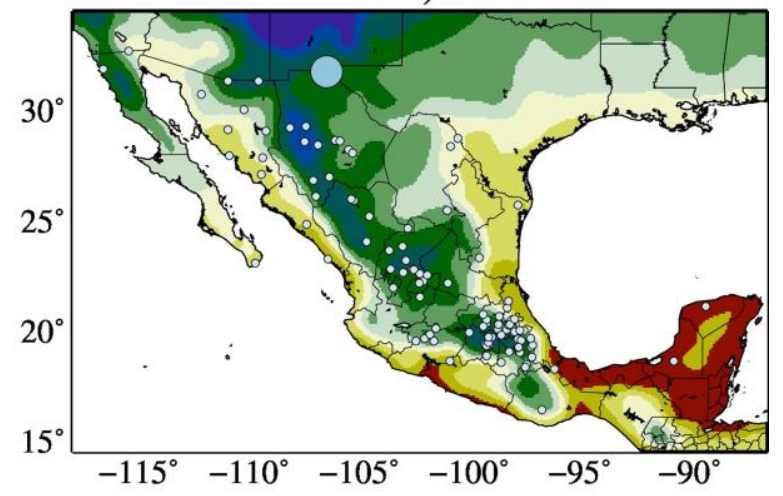

d)



Nota: el tamaño de los círculos, al ser proporcional al número de muertes, puede abarcar municipios aledaños al municipio en el que se registraron las muertes indicadas.

Fuente: elaboración propia.

No se encontró ningún patrón sobresaliente al analizar la distribución espacial de la mortalidad por frío por grupos demográficos; es decir, tanto mujeres (cuadro 6b) como hombres (cuadro 6c), en menores a 5 años (cuadro 6d) o en otros grupos (no mostrado), sigue el patrón general total descrito anteriormente (cuadro 6a).

La región norte registra el mayor número de muertes y su temperatura mínima es la más baja (aprox. $4<T_{\min }<8^{\circ} \mathrm{C}$ ). Sobre el Altiplano Central, casi todas las muertes se encuentran dispersas, pero tienden a ser más pronunciadas cerca de las zonas montañosas de la Sierra Madre Oriental (región fronteriza con el estado de Veracruz); las temperaturas mínimas en esta zona son relativamente frías (aprox. $8^{\circ} \mathrm{C}<T_{\min }<12^{\circ} \mathrm{C}$ ). 
La mortalidad sobre la frontera México-Estados Unidos se acentúa sobre Cuidad Juárez (al cual le corresponde el círculo más grande en el cuadro 6a). Ciudad Juárez registra el mayor número de muertes por frío natural excesivo a nivel nacional, con 119 registradas. Esta ciudad es seguida por Guachochi (Chihuahua), con 66 muertes, y por Hermosillo (Sonora), con 44.

Aunque la región sureste de México (cuadro 6a) no presenta una mortalidad relativamente alta a nivel nacional, la ocurrencia de tales eventos es llamativa porque estas regiones son cálidas (como Tabasco y la Península de Yucatán; $T_{\min }>16^{\circ} \mathrm{C}$ ). En particular, destaca el máximo de mortalidad relativo sobre Los Altos de Chiapas, que aunque tiene mínimo local de temperatura $\left(T_{\min } \sim 15^{\circ} \mathrm{C}\right)$, su vulnerabilidad es característicamente alta (PNUD, 2019). Estos resultados en general son consistentes con diferentes estudios previos (Kempainen y Brunette 2004; Mulcahy y Watts, 2009), pues han documentado que las muertes por exposición al frío excesivo pueden ocurrir durante todas las estaciones del año y en todos los climas, y que tal patrón espacial de la mortalidad puede estar relacionado con la vulnerabilidad de la población (e. g., Milojevic et al., 2016; Singh et al., 2019).

\section{Tendencias en tasas de mortalidad y temperatura}

Sobre el análisis de tendencias, las de tasas de mortalidad negativas (disminución de casos; cuadro 7a) y positivas (incremento de casos; cuadro $7 \mathrm{~b}$ ) presentan patrones similares, pero sólo hay significancia estadística en las de incremento de tasas de mortalidad al oeste de Chihuahua, así como entre Veracruz y San Luis Potosí. Por otro lado, respecto a la temperatura (cuadro 7 a y b), la mayor parte del país ha registrado tendencias estadísticamente significativas de calentamiento (tendencias positivas), las cuales son principalmente notorias sobre las regiones norte y noroeste de México y centro-sur, así como la Península de Yucatán.

Se observa que algunas regiones se han calentado y han disminuido las tasas de mortalidad (ninguna de éstas tiene tendencias estadísticamente significantes; cuadro 7a); pero existe un resultado llamativo: algunas regiones se han calentado y, a la par, han registrado un incremento en las tasas de mortalidad por frío intenso (éstas sí tienen tendencias estadísticamente significantes; cuadro $7 \mathrm{~b}$ ). Una posible explicación es que la aclimatización a un clima más cálido podría incrementar la vulnerabilidad de las personas a eventos extremos fríos (e. g., Medina-Ramon y Schwartz, 2007); eventos que no se reflejan fácilmente al analizar tendencias anuales como en el cuadro 7. Para esclarecer esta idea, sería necesario analizar las tendencias estacionales de temperaturas mínimas en resolución diaria. Tal trabajo, sin embargo, se encuentra fuera de los alcances de este estudio. 
Cuadro 7. Tendencias de temperatura mínima mensual con las tendencias de tasas de mortalidad (cada 100,000 hab.) por frío excesivo (a) negativas y (b) positivas sobre puestas
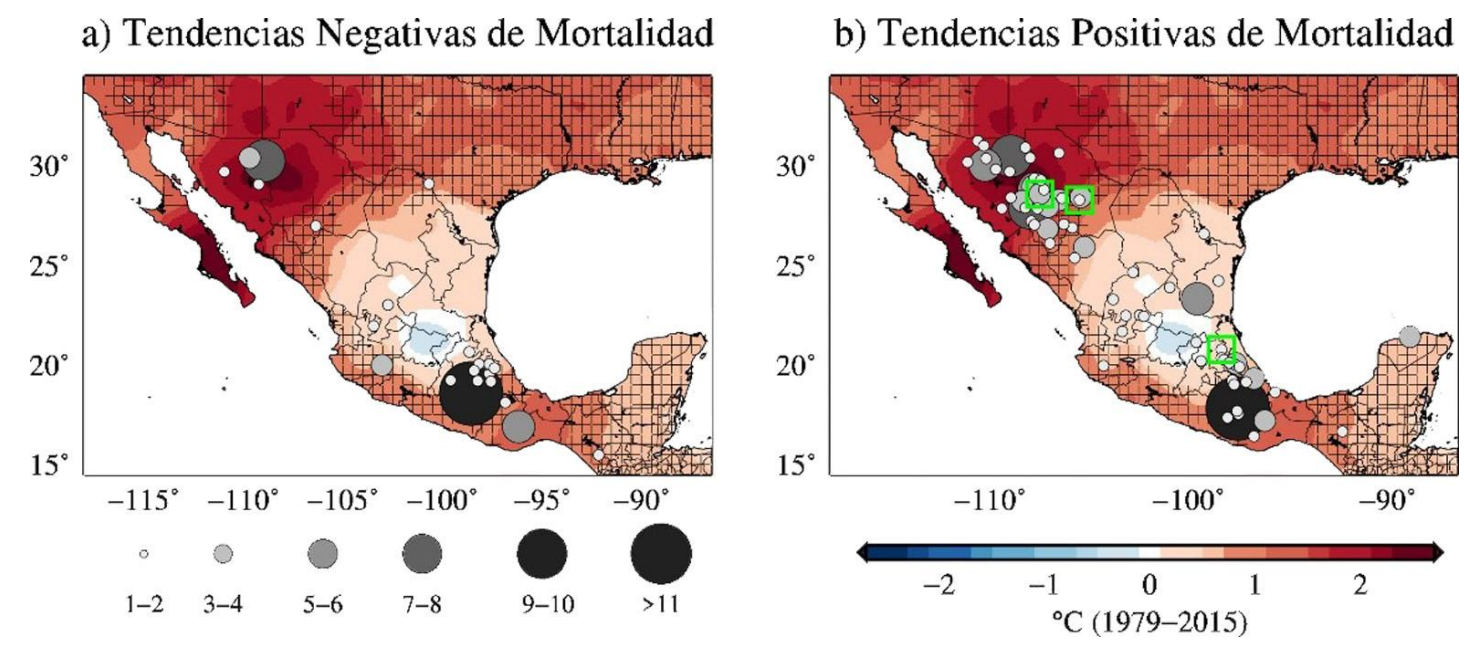

Nota: las tendencias estadísticamente significativas de temperatura se muestran sombreadas; las de mortalidad son el centro de los cuadros color verde. El tamaño de los círculos, al ser proporcional al valor de las tendencias, puede abarcar municipios aledaños al municipio donde se registraron las tendencias indicadas.

Fuente: elaboración propia.

\section{Conclusiones}

En este trabajo, la distribución espacio-temporal de la mortalidad por frío excesivo es investigada dentro de un contexto climático caracterizado por temperaturas mínimas mensuales. En términos generales, esta investigación contribuye a caracterizar el problema general relativo a los impactos del frío excesivo en México bajo la perspectiva de la gestión del riesgo, analizando conjuntamente amenazas y vulnerabilidades. De manera particular, los resultados detallan cuantitativamente los patrones espaciales, temporales y demográficos asociados a la mortalidad por frío intenso.

Mientras que una parte de los resultados documenta coincidencias con el paradigma "a más frío, más muertes por frío intenso", sobre todo en la ubicación de regiones con alta mortalidad, otra parte muestra resultados contrastantes (e. g., una correlación débil entre tasas de mortalidad y temperaturas mínimas). Por ejemplo, con este paradigma es difícil explicar la ocurrencia de muertes por frío durante temporadas y regiones cálidas (sureste de México) o las tendencias de tasas de mortalidad positivas en medio de un clima más cálido. Sin embargo, estas observaciones deben ser analizadas bajo el enfoque del riesgo, bajo el cual las amenazas se combinan con la vulnerabilidad. 
Basados en la existencia de una vulnerabilidad dinámica y compleja, suponemos que una posible explicación es que la aclimatización a un clima más cálido podría incrementar la vulnerabilidad de la población a eventos fríos de corta duración (Medina-Ramon y Schwartz, 2007). Tal hipótesis explicaría parte de las muertes registradas en las temporadas y regiones cálidas; sin embargo, para probarla, se necesitan estudios más detallados sobre el tema, pues los datos aquí analizados, en escalas mensuales, sólo pueden constituir evidencia para su formulación.

Idealmente, los resultados de esta investigación podrían formar parte de una base de datos climática y social que sirviera para el diseño de estrategias de prevención ante el frío intenso. Ello sería importante debido a los altos índices de pobreza (e. g., Cohen y Dechezleprêtre, 2017; PNUD, 2019) que caracterizan las regiones con mayor mortalidad por frío.

\section{Referencias}

Blaikie P., T. Cannon, I. Davis y B. Wisner. (1996). Vulnerabilidad: El Entorno Social, Político y Económico de los Desastres. LA RED: Red de Estudios Sociales en Prevención de Desastres en América Latina. Intermediate Technology Development Group.

Cardona, O. D., et al. (2012). Determinants of risk: Exposure and vulnerability. In: Field, C.B., V. Barros, T.F. Stocker, D. Qin, D.J. Dokken, K.L. Ebi, M.D. Mastrandrea, K.J. Mach, G.-K. Plattner, S.K. Allen, M. Tignor, and P.M. Midgley (Eds.). Managing the Risks of Extreme Events and Disasters to Advance Climate Change Adaptation, Cambridge: Cambridge University Press

Cohen F., y Dechezleprêtre A. (2017). Mortality inequality, temperature and public health provision: evidence from Mexico. Grantham Research Institute on Climate Change and the Environment Working Paper. No. 268. Recuperado de: http://www.lse.ac.uk/GranthamInstitute/publication/mortality-temperature-andpublic-health-provision-evidence-from-mexico/

CRU (2019). CRU TS4.01: Climatic Research Unit (CRU) Time-Series (TS) version 4.01 of high-resolution gridded data of month-by-month variation in climate (Jan. 1901- Dec. 2016). Recuperado de: http://data.ceda.ac.uk/badc/cru/data/cru_ts/cru_ts_4.01/data/

Compeán, R. G. (2013). The death effect of severe climate variability. Procedia Economics and Finance, 5, $182-191$.

De Sherbinin, A., A. Schiller y Pulsipher A. (2007). The vulnerability of global cities to climate hazards. Environment and Urbanization, 19(1), pp. 39-64, https://doi.org/10.1177/0956247807076725

GDIS (2020). Defunciones. Recuperado de: http://www.dgis.salud.gob.mx/contenidos/basesdedatos/da_defunciones_gobmx.html

Githeko A. K., Woodward A. (2003). International consensus on the science of climate and health: the IPCC Third Assessment Report. En: McMichal AJ, Campbell-Lendrum DH, Corvalán CF, Ebi KL et al. (ed.), Climate Change and Human Health, pp 43-60. Geneva: World Health Organization

INEGI (2010). Censos. Recuperado de: https://www.inegi.org.mx/programas/ccpv/2010/default.html

INEGI (2011). $\quad$ Estadísticas Vitales. $\quad$ Recuperado de: https://www.inegi.org.mx/rnm/index.php/catalog/57/dataprocessing

INEGI (2019). Mortalidad. Recuperado de: https://www.inegi.org.mx/programas/mortalidad/

IPCC (2012). Managing the Risks of Extreme Events and Disasters to Advance Climate Change Adaptation. Cambridge University Press. Recuperado de: https://www.ipcc.ch/report/managing-the-risks-of-extremeevents-and-disasters-to-advance-climate-change-adaptation/

Jáuregui Díaz, J. A. J., Sánchez, M. D. J. Á., y Cabañas, R. T. (2020). Cambios en la Mortalidad por Eventos Climáticos Extremos en México entre el 2000 y 2015. REDER, 4(1), 80-94. 


\section{AÑo 23, 2021-1 ENERO-JUNIO E-ISSN 2594-102X PÁGS.73-87 DUHFï}

Kempainen R., y Brunette D., (2004). The evaluation and management of accidental hypothermia. Respir Care. 49 (2), pp. 192-205.

Knaul Felicia, González-Pier Eduardo, Gómez-Dantés Octavio (2012). The quest for universal health coverage: achieving social protection for all in Mexico. The Lancet. 380 (9849): pp. 1259-1279.

McMichael, A. et al. (2008). International Study of Temperature, Heat and Urban Mortality: The 'ISOTHURM' Project. International Journal of Epidemiology, 37(5), pp. 1121-1131.

Magaña, V. (2013). Guía Metodológica para la Evaluación de la Vulnerabilidad ante Cambio Climático. Ciudad de México: INECC, SEMARNAT. Recuperado de: http://climasaludal.org/resources/images/public/avirtuales/cuarta_conferencia/140923_guia_met odologica.pdf

Medina-Ramon, M. y J. Schwartz. (2007). Temperature, Temperature Extremes, and Mortality: A Study of Acclimatization and Effect Modification in 50 United States Cities, Occupational and Environmental Medicine. 64(12), pp. 827-33. DOI: 10.1136/oem.2007.033175

Milojevic, A., Armstrong, B. G., Gasparrini, A., Bohnenstengel, S. I., Barratt, B., y Wilkinson, P. (2016). Methods to estimate acclimatization to urban heat island effects on heat-and cold-related mortality. Environmental health perspectives, 124(7), 1016-1022.

Mulcahy, A. y Watts M. (2019). Accidental Hypothermia: An Evidence-Based Approach. Emergency Medicine Practice, 11(1).

Neri, C., y Magaña, V. (2016). Estimation of vulnerability and risk to meteorological drought in Mexico. Weather, Climate, and Society, 8(2), 95-110

Nixdorf-Miller A., Hunsaker D. M., Hunsaker J. C. (2006). Hypothermia and hyperthermia medicolegal investigation of morbidity and mortality from exposure to environmental temperature extremes. Arch Pathol Lab Med. 130() p. 1297-1304.

OMS (2020). Clasificación Internacional de Enfermedades (CIE). Recuperado de: https://www.paho.org/hq/index.php?option=com_content\&view=article\&id=3561:2010clasificacion-internacional-enfermedades-cie\&Itemid $=2560 \& l a n g=e s$

PNUD (2019). Informe de Desarrollo Humano Municipal 2010-2015: Transformando México desde lo local. Recuperado de:

https://www.mx.undp.org/content/dam/mexico/docs/Publicaciones/PublicacionesReduccionPob reza/InformesDesarrolloHumano/idhmunicipal20102015/Informe\%20IDHMunicipal-completo.pdf

Riojas Rodríguez, H., Hurtado Díaz M., Idrovo Velandia J., y Vázquez Grameix H. (2006). Estudio diagnóstico sobre los efectos del cambio climático en la salud humana de la población en México: Informe final, 33 pp., Instituto Nacional de Ecología, Instituto Nacional de Salud Pública. Recuperado de: http://www2.inecc.gob.mx/descargas/cclimatico/e2006h.pdf

Santer, B. D., Wigley, T. M. L., Boyle J. S., Gaffen, D. J., Hnilo, J. J., Nychka, D., Parker, D. E., y Taylor K. E. (2000). Statistical significant of trends and trend differences in layer-average atmospheric temperature time series. Journal of Geophysical Research, (105), D6, pp. 7337-7356.

Singh A., Stead T. G., Mangal R., Banerjee P., Ganti L. (2019). Severe Hypothermia in the Sunshine State. Cureus, 11(7): e5088. https://dx.doi.org/10.7759\%2Fcureus.5088

SINAIS. (2018). Estadísticas. Recuperado de: http://www.dgis.salud.gob.mx/contenidos/sinais/estadisticas.html

Tabor, K., y Williams, J. W. (2010). Globally downscaled climate projections for assessing the conservation impacts of climate change. Ecological Applications, 20(2), pp. 554-565.

Taylor A. J., McGwin G. Jr., Davis G. G., Brisie R. M., Holley T. D., y Rue L. W. III (2001). Hypothermia deaths in Jefferson County Alabama. Hypothermia deaths in Jefferson County, Alabama, Injury Prevention. (7), pp. 141-145.

Vargas, N., y Magaña, V. (2020). Warm Spells and Climate Risk to Human Health in the Mexico City Metropolitan Area. Weather, Climate, and Society, 12(3), 351-365. 